\title{
ARTICLE
}

Received 23 Oct 2012 | Accepted 30 Jan 2013 | Published 5 Mar $2013 \quad$ DOI: 10.1038/ncomms2564

\section{High-pressure synthesis of a polyethylene/zeolite nano-composite material}

Mario Santoro ${ }^{1}$, Federico A. Gorelli ${ }^{2,3}$, Roberto Bini ${ }^{2,4}$, Julien Haines ${ }^{5}$ \& Arie van der Lee ${ }^{6}$

Meso/micro-porous solids, such as zeolites, are complex materials used in an impressive range of applications. Here we photo-polymerized ethylene using non-catalytic high-pressure techniques at $0.5-1.5 \mathrm{GPa}$ under ultraviolet $(351-364 \mathrm{~nm}$ ) irradiation on a sub-nanometre scale in the channels of a pure $\mathrm{SiO}_{2}$ zeolite, silicalite, to obtain a unique nano-composite material with drastically modified mechanical properties. The structure obtained contains single polyethylene chains, which adapt very well to the confining channels as shown by optical spectroscopy and X-ray diffraction. The formation of this nano-composite results in significant increases in bulk modulus and density, and the thermal expansion coefficient changes sign from negative to positive with respect to silicalite. Mechanical properties may thus be tuned by varying the amount of polymerized ethylene. Our findings could allow the high-pressure, catalyst-free synthesis of a unique generation of technological, functional materials based on simple hydrocarbons polymerized in confining meso/micro-porous solids.

\footnotetext{
${ }^{1}$ Istituto di Fisica Applicata N. Carrara, IFAC-CNR, 50019 Sesto Fiorentino, Firenze, Italy. ${ }^{2}$ European Laboratory for non Linear Spectroscopy, LENS, 50019 Sesto Fiorentino, Firenze, Italy. ${ }^{3}$ Istituto per i Processi Chimico Fisici, IPCF-CNR, UOS Roma, 00185 Roma, Italy. ${ }^{4}$ Dipartimento di Chimica dell'Università di Firenze, 50019 Sesto Fiorentino, Firenze, Italy. ${ }^{5}$ Institut Charles Gerhardt Montpellier, UMR 5253 CNRS, Equipe C2M, Université Montpellier 2, 34095 Montpellier, France. ${ }^{6}$ Institut Européen des Membranes, UMR-CNRS 5635, Université Montpellier 2, 34095 Montpellier, France. Correspondence and requests for materials should be addressed to M.S. (email: m.santoro@ifac.cnr.it or santoro@lens.unifi.it).
} 
$\mathrm{M}$ eso/micro-porous materials can be considered as very small chemical laboratories, where chemical reactions are induced and controlled on a small scale down to just a few angstroms. Their large-scale applications are, among others, fine gas separation, gas storage and catalysis ${ }^{1-9}$. More emerging applications of these materials, once properly functionalized, are also in sight in diverse fields such as nano-electronics, nanosensoring and nano-photonics ${ }^{10,11}$. A very intriguing aspect of ordered nano- and subnano-porous solids is the ability to drive the formation of confined polymers with remarkable physical properties (see refs 12-17 and other references therein). Polymerization is usually performed by complex chemical methods including the use of catalysts and radical initiators, which are either supplied by the wall of the nano-pores or are added to the pores together with the reactants. This type of chemistry was used, for instance, to encapsulate conducting filaments of polyaniline ${ }^{18}$ and graphite-type carbon wires ${ }^{19}$ in the 3-nm-wide ordered hexagonal channel system of the porous aluminosilicate MCM-41. Crystalline linear polyethylene (PE) nano-fibers have also been produced by catalysed polymerization and extrusion through meso-porous silica ${ }^{20}$. The goal of this study is to push the effect of confinement on polymerizing matter down to the sub-nanometre scale, while avoiding any catalytic effects from the pore walls to better understand the effect of confinement per se and to fully characterize these materials from a structural point of view. Unique nano-composite materials could be synthesized in this way. A strong advantage of highpressure polymerization techniques with respect to more conventional catalytic protocols is precisely that these techniques allow the use of catalysts and radical initiators to be avoided $^{21-23}$, which is indispensable to determine the intrinsic role of spatial confinement on polymerizing matter. Indeed, pressure is the most efficient tool to reduce intermolecular distances, thereby permitting continuous tuning of the corresponding interactions and allowing the chemical reactions to occur, which are in some cases enhanced by ultraviolet (UV) light irradiation, when a critical intermolecular distance is approached in this way ${ }^{24}$. Another strong advantage of the high-pressure methodology in synthesizing composite materials is that once these procedures are scaled up to provide sufficiently large amounts of product material, they could represent a true, novel 'green chemistry' route to industrial chemical synthesis, as the use of additional and polluting compounds such as the catalysts and radical initiators is avoided.

The best candidate as the host material for our purpose to synthesize a nano-composite material can be found among zeolites. These materials are micro-porous, crystalline, inorganic solids made of corner-sharing $\mathrm{SiO}_{4}$ and $\mathrm{AlO}_{4}$ tetrahedra with strong acid character and catalytic properties. At one end of this class, there is silicalite, which is an electrically neutral, noncatalytic pure $\mathrm{SiO}_{2}$ zeolite. Silicalite is characterized by a framework of 4-, 5-, 6- and 10-membered rings of cornersharing $\mathrm{SiO}_{4}$ tetrahedra forming interconnected, mutually orthogonal straight and sinusoidal $5.5 \AA$ diameter channels (see ref. 25 and references therein). Silicalite can easily adsorb simple molecules, such as $\mathrm{Ar}, \mathrm{CO}_{2}$ refs 26-28), and straight n-alkanes (up to $n=10$ ), and, interestingly, computer simulations indicate that $n$-alkane chains easily adapt to the channels ${ }^{29,30}$. We then chose ethylene as a benchmark, simple hydrocarbon to undergo polymerization in the sub-nano channels of silicalite with the aim of producing a unique nano-composite material. To our knowledge, ethylene was never polymerized in a nano-confining host material, neither by catalytic nor by non-catalytic routes. Polymerization was induced by applying pressures of $0.5-1.5 \mathrm{GPa}$ to the confined reactant and using UV irradiation (351-364 nm) as an optical catalyst, which we have already shown to be efficient conditions for polymerizing bulk ethylene and other simple hydrocarbons ${ }^{21-23}$. The composite was characterized by optical spectroscopy, and single-crystal and powder X-ray diffraction (XRD). The structure obtained contains single PE chains, which adapt very well to the confining channels and strongly modify the mechanical properties of the host zeolite. Here and in the following the nano-composite product material will be referred to as PESIL, from PE and SIL (silicalite).

\section{Results}

Synthesis of PESIL. Supercritical fluid ethylene was loaded in a diamond anvil cell (DAC) and compressed to $0.5-1.5 \mathrm{GPa}$, at room temperature, together with either polycrystalline or singlecrystal silicalite-1F. A total of four samples were prepared in this way. In the polycrystalline case, finely ground silicalite powder almost completely filled the sample thickness $(40-80 \mu \mathrm{m})$, so that the vast majority of the loaded ethylene occupied the micropores, and only a small remaining amount was found in the interstitial spaces as bulk $\mathrm{C}_{2} \mathrm{H}_{4}$. In the second case, a single crystal of typically $40 \times 40 \times 80 \mu \mathrm{m}^{3}$ was placed in the gasket that was then filled with ethylene, which entered into the pores and also formed a bulk, surrounding domain. Ethylene completely filled the pores of silicalite, as has previously been found for other small molecules and atoms such as $\mathrm{Ar}$ and $\mathrm{CO}_{2}$ (refs 26-28). In fact, gas adsorption in silicalite reaches saturation at typical pressures well below the liquid-vapor transition pressure ${ }^{30}$, whereas our compressed fluid is about $25 \%$ denser than the liquid phase. The Raman active $\mathrm{C}=\mathrm{C}$ stretching mode of ethylene at about $1,628 \mathrm{~cm}^{-1}$ shows a clear signature of the micro-confined molecules (Fig. 1). The bands of bulk and confined ethylene are

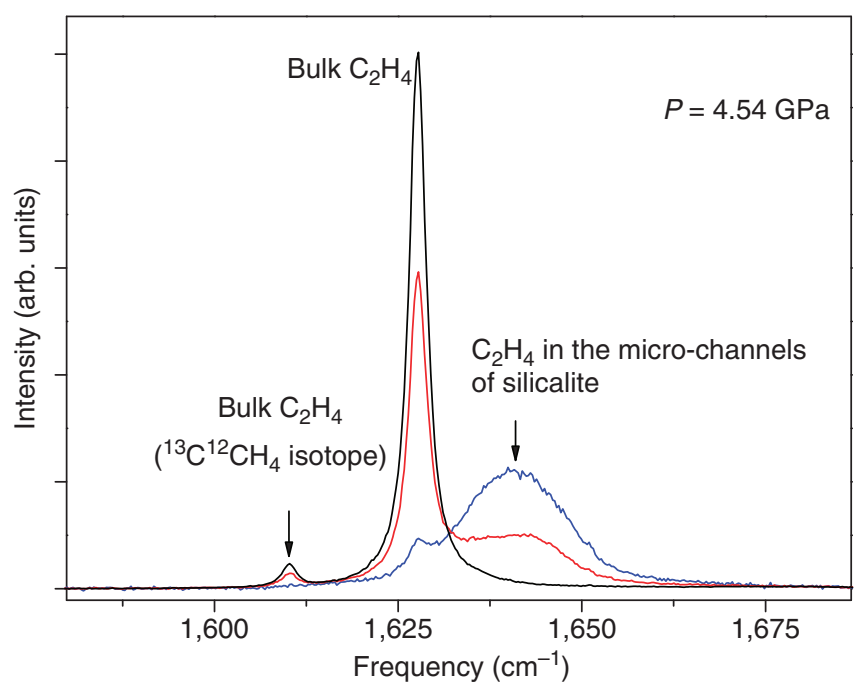

Figure 1 | Raman spectra of the $\mathbf{C}=\mathbf{C}$ stretching line of ethylene. Measurement was performed at $4.54 \mathrm{GPa}$ on a mixture of $\mathrm{C}_{2} \mathrm{H}_{4}$ and silicalite-1F single crystals. (i) Black spectrum, measured on bulk ethylene (thickness of about $40 \mu \mathrm{m}$ ); (ii) red spectrum, measured on a silicalite crystal with bulk ethylene on the top $\left(\mathrm{C}_{2} \mathrm{H}_{4}\right.$ thickness of about $\left.10 \mu \mathrm{m}\right)$; (iii) blue spectrum, measured on the top of a silicalite crystal, which is nearly bridging the two diamond anvils $\left(\mathrm{C}_{2} \mathrm{H}_{4}\right.$ thickness of less than $\left.1 \mu \mathrm{m}\right)$. Sharp peaks at about $1,628 \mathrm{~cm}^{-1}$ and $1,610 \mathrm{~cm}^{-1}:{ }^{12} \mathrm{C}={ }^{12} \mathrm{C}$ and ${ }^{13} \mathrm{C}={ }^{12} \mathrm{C}$ stretching lines of bulk $\mathrm{C}_{2} \mathrm{H}_{4}$, respectively. Broad peak at about $1,641 \mathrm{~cm}^{-1}$. ${ }^{12} \mathrm{C}={ }^{12} \mathrm{C}$ stretching band of ethylene confined in the micro-channels of silicalite. The three spectra have been normalized to the same integrated intensity. Ethylene is in solid phase I and we did not observe spontaneous chemical reactions at the typical time scale (1-2h) of the Raman investigation (see also in ref. 31). 
split by about $8 \%$ and that of the confined molecules is much broader. This indicates the presence of a moderate interaction between $\mathrm{C}_{2} \mathrm{H}_{4}$ and the silicalite wall surface, which did not lead to significant chemical reactivity. The polymerization of ethylene was then performed at $0.5-1.5 \mathrm{GPa}$ under UV irradiation (CW $\mathrm{Ar}^{+}$laser, at $351-364 \mathrm{~nm}$ ) for a few hours with typical power density of $1.6-3.2 \mathrm{~W} \mathrm{~mm}^{-2}$. Polymerization and the obtained PESIL were investigated by Raman and IR absorption spectroscopy, and powder and single-crystal XRD.

Characterization of PESIL by IR spectroscopy. IR spectroscopy shows that ethylene undergoes polymerization giving rise to almost $100 \%$ conversion of the reactant over a few hours (Fig. 2a). The sample was then recovered at ambient pressure and further investigated (Fig. 2b). The IR spectrum provides important information on the structure of the confined PE as seen, for instance, in the spectral region of the intense $\mathrm{CH}_{2}$ bending mode doublet at $1,463-1,473 \mathrm{~cm}^{-1}$. The structure of the doublet is characteristic of an ordered orthorhombic crystal (Pnam, $Z=2$ ). In the case of high-pressure, UV-assisted polymerization of bulk ethylene, this doublet is by far the dominant feature between 1,300 and $1,500 \mathrm{~cm}^{-1}$ (ref. 21). In our case, when either part or almost the full sample region is occupied by silicalite powder, we observe in the middle of the sharp doublet, due to bulk PE, a strong and broad single-chain bending peak at $1,468 \mathrm{~cm}^{-1}$ with shoulders at 1,458 and $1,445 \mathrm{~cm}^{-1}$, and two broad wagging mode bands at 1,353 and $1,370 \mathrm{~cm}^{-1}$. The shoulders and the wagging peaks, which are very weak in the bulk polymer ${ }^{21,31}$, are entirely due to gauche defects. All these peaks correspond to disordered $\mathrm{PE}$ with gauche defects among others, which we assign to confined PE in the micro-channels of silicalite. It is thus evident that some strain is imposed on the polymeric chains due to the high degree of confinement. In addition, the relatively sharp, weak peak at $1,379 \mathrm{~cm}^{-1}$ is due to the methyl groups of confined PE. This implies the presence of a few relatively short branched chains, which are probably related to crystalline defects in silicalite.

Characterization of PESIL by XRD. While optical spectroscopies give important information on the structure of PESIL, XRD using single-crystal data obtained from a typical sample recovered at ambient pressure is the most suitable technique to determine its crystal structure (Table 1 and Supplementary Tables S1 and S2). Starting from the Pnma structural model for the $\mathrm{SiO}_{2}$ framework, the $\mathrm{C}$ atoms were located from Fourier difference maps giving a total of $68 \mathrm{C}$ atoms per unit cell. This indicates that before polymerization silicalite contained 34 molecules of ethylene per unit cell, which is significantly higher than the 11 molecules per unit cell typically obtained by gas adsorption ${ }^{32}$. The resulting structure is unique (Fig. 3 and Supplementary Fig. S1) in terms of the arrangement of single $\mathrm{PE}$ chains within the confining channels. Four single, ideally infinite PE chains form inside the unit cell of silicalite, each having $17 \mathrm{C}$ atoms per unit cell, and adapt fairly well to the shape of the 10-membered ring channels, whereas the smaller channels do not contain PE, because of steric hindrance. However, these chains are affected by a high degree of strain as a result of the nano-confinement. Two straight, zig-zag chains lie in the straight channels along $\mathbf{b}$ with the carbon atoms remarkably displaced from the plane containing what should be the ideal zig-zag skeletal $\mathrm{C}$ chain ${ }^{33}$. The other two chains lie in the two sinusoidal channels in the ac plane. In this case, not only do the carbon atoms deviate from the skeletal plane, but the chains are also sinusoidal to follow the sinusoidal shape of the confining channels. Importantly, there are no interconnections between the two types of polymeric chains. Confined PE is not commensurate with the host channels, because of the strong
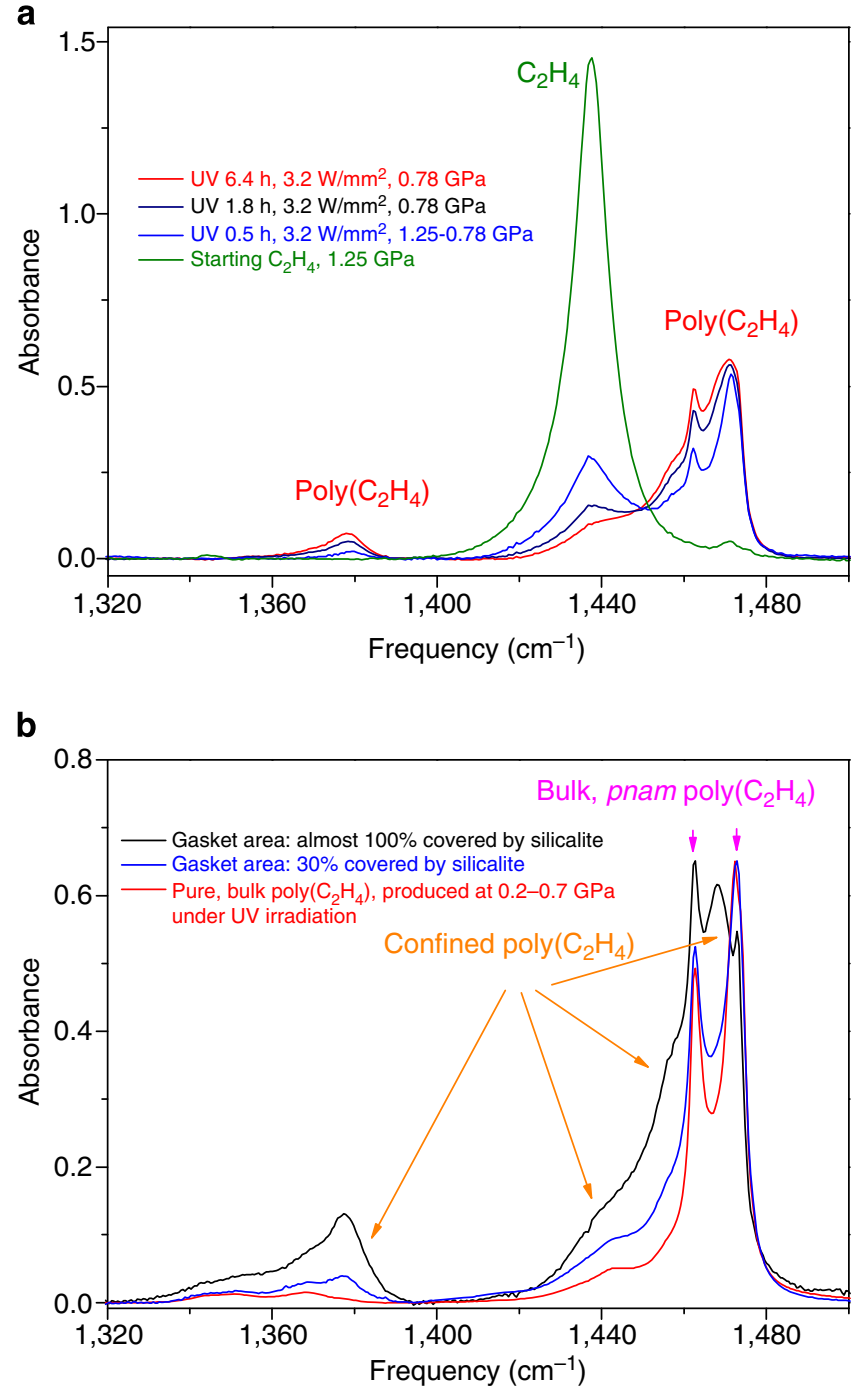

Figure 2 | IR absorption spectra in a selected frequency region showing the formation of confined and bulk polyethylene. (a) The sample region is almost fully occupied by silicalite powder. Pressures: 0.78-1.25 GPa. Olive: starting sample showing the strong $\mathrm{CH}_{2}$ bending peak of ethylene at $1,437 \mathrm{~cm}^{-1}$ and a very small amount of the corresponding polyethylene peak at $1,470 \mathrm{~cm}^{-1}$ due to a very small, accidental initial laser irradiation. Blue, navy and red: the same sample after $0.5,1.8$ and $6.4 \mathrm{~h}$ of UV irradiation, respectively, showing the progressive formation of polyethylene at the expense of ethylene. (b) Final (recovered and ambient pressure) mixtures of polyethylene and silicalite powder with the silicalite occupying almost the full sample thickness and about 30\% (blue) and 100\% (black) of the sample area exposed to the incoming IR beam. Red: a pure polyethylene sample obtained under pressure and UV irradiation ${ }^{21}$. The sharp doublet at $1,463-1,473 \mathrm{~cm}^{-1}$ is due to bulk polyethylene, while all the other peaks are mainly due to highly strained, confined polyethylene chains in the micro-channels of silicalite. The three spectra are normalized to the same peak absorbance at $1,450-1,480 \mathrm{~cm}^{-1}$.

strain-related disorder. The large atomic displacement parameters (Supplementary Table S2) can be expected to correspond to static distributions of the occupation of the carbon atoms in the pore and thus to translational and some rotational static disorder of the PE chains. The presence of defects in confined PE, as indicated by IR spectroscopy, is thereby confirmed by the structural analysis. Another spectacular aspect of PESIL is the remarkable change found in the structure of the SIL host with 
respect to that of pure, ambient pressure monoclinic silicalite. As in the case of silicalite filled by Ar and $\mathrm{CO}_{2}$ under pressure ${ }^{26}$, the unit cell metric is now almost pseudo-tetragonal with the $a$ and $b$ lattice parameters only differing by $0.1 \%$ and the PE-filled silicalite cell volume is about $2 \%$ larger than that of the empty pure $\mathrm{SiO}_{2}$ cell. The volume thermal expansion coefficient was found to be $7 \times 10^{-5} \mathrm{~K}^{-1}$ based on variable temperature XRD measurements. This is also surprising as filling with PE has the effect of changing the sign of the thermal expansion coefficient of

\section{Table I | Single-crystal data and bulk modulus of PESIL $\left(\left(\mathrm{SiO}_{2}\right)_{96}\left(\mathrm{C}_{2} \mathrm{H}_{4}\right)_{34}\right.$, space group Pnma).}

\begin{tabular}{lcc} 
Single crystal & $\boldsymbol{T}=\mathbf{2 9 3} \mathbf{K}$ & $\boldsymbol{T}=\mathbf{1 5 0} \mathbf{K}$ \\
\hline Unit cell parameters, density and volume thermal expansion coefficient \\
a $(\AA)$ & $20.100(2)$ & $20.057(17)$ \\
b $(\AA)$ & $20.071(5)$ & $19.995(7)$ \\
c $(\AA)$ & $13.469(1)$ & $13.414(3)$ \\
V $\left(\AA^{3}\right)$ & $5,434(2)$ & $5,380(4)$ \\
Density $\left(\mathrm{g} \mathrm{cm}^{-3}\right)$ & $2.054(1) ;$ & $2.075(2)$ \\
& 2.648 for $\alpha$-quartz \\
Volume thermal expansion & $7(1) \times 10^{-5} ;$ \\
$\left(\mathrm{K}^{-1}\right)$ & $3.6 \times 10^{-5}$ & \\
& for $\alpha$-quartz & \\
&
\end{tabular}

\section{Powder}

Mechanical properties

Bulk modulus (GPa) $26.7(2.0) ; 37$

for $\alpha$-quartz

Corresponding values for $\alpha$-quartz are given $35,41,42$ for comparison. Structural data obtained from refinement of the structure of the single-crystal sample at ambient pressure and temperature are reported in Supplementary Tables S1 and S2.

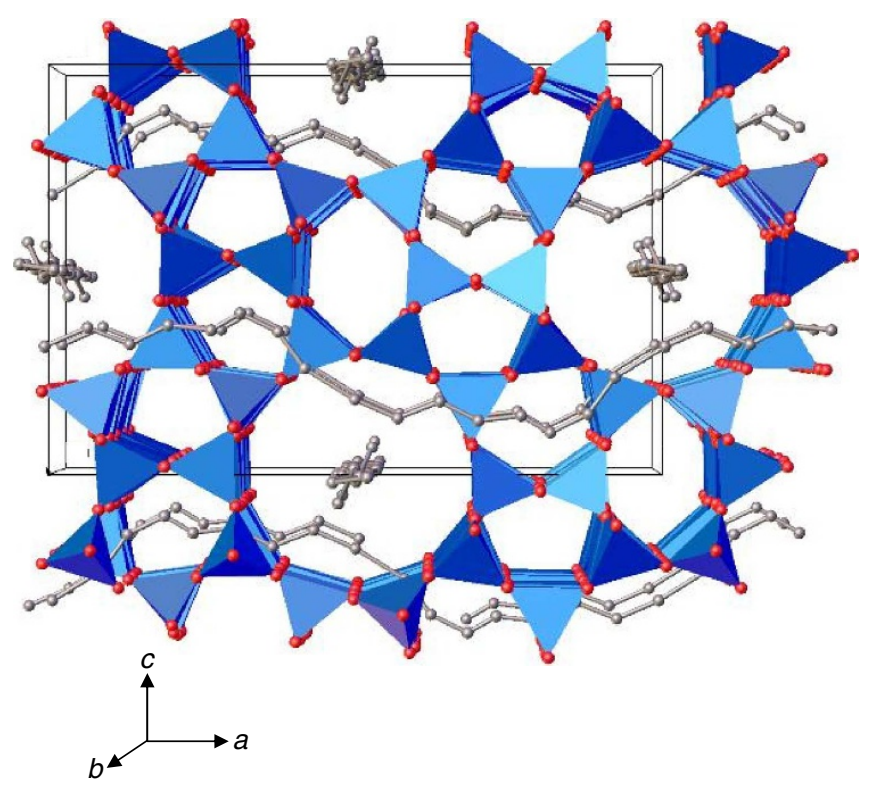

Figure 3 | Structure of PESIL (space group Pnma) obtained by singlecrystal XRD. Blue polyhedra: $\mathrm{SiO}_{4}$ tetrahedra; red spheres: $\mathrm{O}$ atoms; grey spheres: $\mathrm{C}$ atoms. Hydrogen atoms are not shown. Four ideally infinite polyethylene chains form in the unit cell of silicalite: two straight ones along the $b$ axis and two sinusoidal ones in the $a c$ plane. There are no interconnections between the two types of polymeric chains. The polymer chains are severely strained and not commensurate with silicalite. See also Supplementary Figure S1. orthorhombic SIL from a negative ${ }^{8,9}$ to a normal positive value, which is close to twice that of quartz and one order of magnitude lower than that of ultra high molecular-weight pure $\mathrm{PE}$ (UHMWPE).

The mechanical properties of PESIL were determined by measuring the XRD patterns as a function of pressure at room temperature on the polycrystalline sample. The equation of state (EOS) was obtained along with a test of resistance against pressure-induced amorphization ${ }^{25}$ (Fig. 4). The XRD pattern of silicalite strongly changes when it is filled by ethylene under pressure, as observed before inducing polymerization (Fig. 4a): the low angle peaks below $5^{\circ}$ remarkably weaken with respect to those above $8^{\circ}$ as a result of changes in structure factor due to the presence of $\mathrm{C}_{2} \mathrm{H}_{4}$. This trend was already observed in the case of silicalite filled with $\mathrm{CO}_{2}$ and $\mathrm{Ar}$ at high pressures and is another proof that the host micro-channels are filled with guest molecules ${ }^{26,27}$. After polymerization, PESIL was investigated under pressure up to $24 \mathrm{GPa}$. The XRD pattern still exhibits Bragg peaks at the maximum pressure, thereby showing that
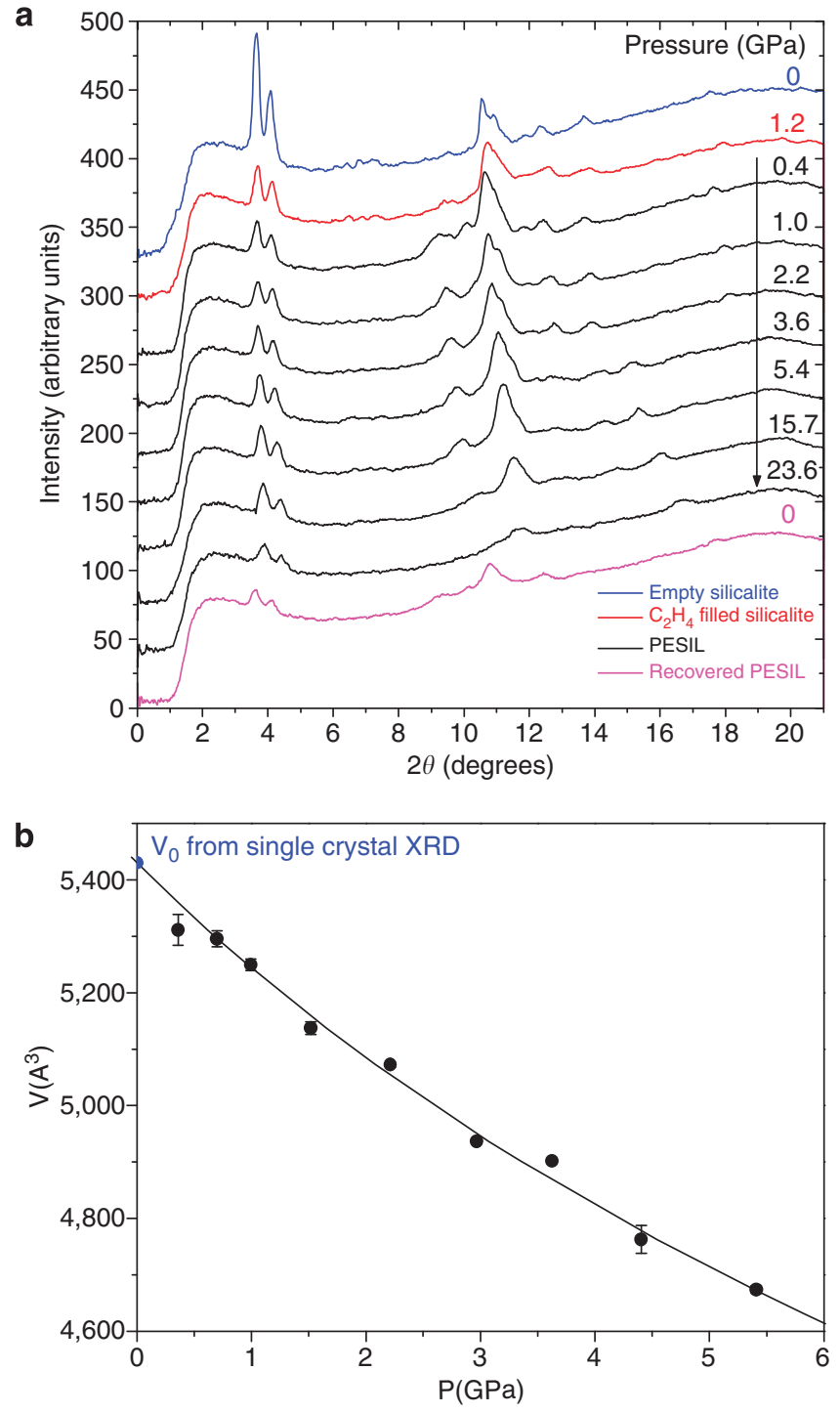

Figure 4 | Powder XRD on PESIL upon pressure increase. (a) XRD patterns of empty silicalite-1F (blue), ethylene-filled silicalite before polymerization (red), PESIL under pressure (black) and PESIL recovered at ambient pressure after compression (magenta); (b) equation of state: unit cell volume data versus pressure. 
pressure-induced amorphization of the host silicalite, which occurs progressively in pure silicalite and is complete at about $8 \mathrm{GPa}$, is prevented by the encapsulated $\mathrm{PE}$, which is similar to what was also observed with $\mathrm{CO}_{2}$ and $\mathrm{Ar}$ as guest species ${ }^{26}$. An accurate EOS is obtained upon compression up to $6 \mathrm{GPa}$ (Fig. $4 \mathrm{~b}$ and Table 1), above which PESIL is affected by strong metastabilities, which are probably due to the non-hydrostatic compression. Silicalite-1-F is highly compressible, with a bulk modulus $\left(B_{0}\right)$ of $13.6 \mathrm{GPa}^{25}$. Pressure-volume data for PESIL can be fitted to a third order Birch-Murnagam $\operatorname{EOS}^{34}$ with a $B_{0}$ value of 26.7(2.0) GPa and the first derivative $\left(B_{0}{ }^{\prime}\right)$ of 4 (Table 1). The encapsulated PE in PESIL thus remarkably increases the bulk modulus to a value, which is closer to that of $\alpha$-quartz ${ }^{35}$, as in the case of $\mathrm{CO}_{2}$ - and Ar-filled silicalite ${ }^{26}$. In addition, the density of PESIL is $2.054(1) \mathrm{g} \mathrm{cm}^{-3}$, which is lower than that of $\alpha$-quartz by about $22 \%$, mainly because it is a $\mathrm{SiO}_{2} / \mathrm{C}_{\mathrm{n}} \mathrm{H}_{2 \mathrm{n}}$ composite material.

\section{Discussion}

High-pressure techniques permitted PESIL to be synthesized without catalysts producing a unique nano-composite material made of a pure $\mathrm{SiO}_{2}$ zeolite filled by single PE chains. The new material was recovered at ambient pressure. PESIL is crystalline, although the polymeric chains are strained and disordered as a result of nano-confinement. In fact, the polymers are strained in such a way to adapt fairly well to the confining channels of silicalite. PESIL exhibits interesting mechanical properties: (i) it is only $28 \%$ softer than $\alpha$-quartz, but stiffer than silicalite and UHMWPE by about a factor of 2 and 5, respectively; (ii) it exhibits a volume thermal expansion coefficient close to twice that of $\alpha$-quartz, but which is lower than that of UHMWPE by one order of magnitude; and (iii) it is $22 \%$ less dense than $\alpha$ quartz, whereas it is $15 \%$ denser than silicalite and about a factor of 2.2 denser than UHMWPE. It is particularly interesting that the polymerization of ethylene in silicalite changes the sign of the thermal expansion coefficient from negative to positive. The index of refraction of PESIL could also exhibit intermediate values between those of silicalite and quartz. In principle, PESIL or similar composite materials can be produced with a variety of well-tailored mechanical properties, which could be tuned either by changing the amount of ethylene polymerized in the microchannels of silicalite or by changing the host zeolite. Particularly, a zero thermal expansion version of PESIL could be synthesized. These findings, together with the moderately high pressures needed for the synthesis of PESIL, could open the way for the large-scale production of a new variety of composite materials with interesting technological applications as supports for optics in aerospace devices among others. Even more sophisticated applications could be in sight. The potential high-pressure, catalyst-free synthesis of nano-composite materials similar to PESIL, but based on conducting polymers along with the potential high-pressure functionalization of zeolite micro-channels could open the way to an entirely new class of micro/nanostructured materials uniquely suited for advanced electronic and photonic applications. For instance, high-pressure polymerization could lead to the incorporation of conducting polymers, such as polyacetylene, which would be well protected in the zeolite matrices and are not chemically stable otherwise. This would in turn result in the production of novel, ideal conducting organic/ inorganic composite materials.

\section{Methods}

Optical spectroscopy methods. IR spectra were measured using a Bruker IFS- 120 HR Fourier transform infrared spectrometer equipped with an optical beam condenser based on ellipsoidal mirrors ${ }^{36}$. Raman spectra were performed using the $647.1-\mathrm{nm}$ line of a $\mathrm{Kr}^{+}$laser as the excitation source. Backscattering geometry was used with X20 Mitutoyo micro-objective, and the signal was detected by an Acton/ SpectraPro 2500i single monochromator, equipped with a CCD detector (Princeton Instruments Spec-10:100 BR).

Powder XRD methods. Angle dispersive, powder XRD patterns were obtained using a Mo micro-source $(\lambda=0.71073 \AA)$, equipped with multilayered focusing mirrors (Xenocs-GeniX Mo Small Spot). The final X-ray spots on the sample and beam divergence were $100 \mu \mathrm{m}$ and $0.2^{\circ}$, respectively. The diffracted signal was detected by a CCD detector (PI-SCX 4300). Typical integration time for each pattern was $1 \mathrm{~h}$. The diffraction patterns were then analysed and integrated using the FIT2D program ${ }^{37}$.

Single-crystal XRD methods. Single-crystal XRD measurements were performed using $\mathrm{Cu} \mathrm{K}$ radiation $(\lambda=1.54185 \AA)$ with an Agilent Gemini Diffractometer equipped with a Sapphire CCD detector at $70.00 \mathrm{~mm}$. The single crystal was studied directly in the stainless steel gasket after the high-pressure synthesis. Low temperatures were obtained by cooling the sample in a cold nitrogen jet using an Oxford Instruments Cryojet device. Data collection, indexing and reduction were performed using CrysalisPro (Agilent Technologies). Absorption corrections for the crystal and the bulk PE and gasket shadowing corrections implemented in the software package Absorb 6.0 (ref. 38) were performed. Structure refinements were performed using SHELXL97 (ref. 39). Twinning defined by a twofold twin axis along the [110] direction was taken into account. Due to the high degree of disorder in the PE chains, constraints were added for the $\mathrm{C}$-C distances and the isotropic atomic displacement parameters (ADPs) of the different sets of $\mathrm{C}$ atoms on general positions were constrained to be equal.

High-pressure methods. DACs were used for the high-pressure experiments, with typical sample diameter and thickness of 250 and $30-80 \mu \mathrm{m}$, respectively. Ethylene (purity 99\% from Rivoira) was loaded in the DAC cryogenically in the liquid phase at $-20^{\circ} \mathrm{C}$ and 30 bar together with powdered or single crystal, hydrophobic hydrogen-free silicalite-1-F (SOMEZ, France). A ruby chip was put in the sample chamber for pressure measurements, using the ruby fluorescence method ${ }^{40}$.

\section{References}

1. Mumpton, F. A. La roca magica: uses of natural zeolites in agriculture and industry. Proc. Natl Acad. Sci. USA 96, 3463-3470 (1999).

2. Fachini, A. \& Vasconcelos, M. T. Effects of zeolites on cultures of marine micro-algae-a brief review. Environ. Sci. Pollut. Res. Int. 13, 414-417 (2006)

3. Cejka, C., van Bekkum, H., Corma, A. \& Schueth, F. Introduction to Zeolite Molecular Sieves 3rd Edn (Elsevier, 2007).

4. Breck, D. W. Zeolite Molecular Sieves: Structure, Chemistry and Use (Wiley, 1974).

5. Auerbach, S. M., Carrado, K. A. \& Dutta, P. K. Handbook of Zeolite Science and Technology (Marcel Dekker, Inc., 2003)

6. Galarneau, A., Di Renzo, F., Fajula, F. \& Vedrine, J. Zeolites and Mesoporous at the Dawn of the 21st Century (Elsevier, 2001)

7. Weitkamp, J. \& Puppe, L. Catalysis and Zeolites: Fundamentals and Applications (Springer-Verlag, 1999).

8. Park, S. H., Große Kunstleve, H., Graetsch, H. \& Gies, H. The thermal expansion of the zeolites MFI, AFI, DIH, DDR, and MTN in their calcined and as synthesized forms. Stud. Surf. Sci. Catal. 105, 1989-1994 (1997).

9. Bhange, D. S. \& Ramaswamy, V. High temperature thermal expansion behavior of silicalite-1 molecular sieve: in situ HTXRD study. Micropor. Mesopor. Mater. 103, 235-242 (2007).

10. Davis, M. Ordered porous materials for emerging applications. Nature 417, 813-821 (2002)

11. Maschmeyer, T., Rey, F., Sankar, G. \& Thomas, J. M. Heterogeneous catalysts obtained by grafting metallocene complexes onto mesoporous silica. Nature 378, 159-162 (1995).

12. Wey, L., Nantao, H. \& Zhang, Y. Synthesis of Polymer-Mesoporous Silica Nanocomposites. Materials 3, 4066-4079 (2010).

13. Lu, Y. et al. Self-assembly of mesoscopically ordered chromatic polydiacetylene/ silica nanocomposites. Nature 410, 913-917 (2001).

14. Tajima, K. \& Aida, T. Controlled polymerizations with constrained geometries Chem. Comm. 2000, 2399-2412 (2000).

15. Uemura, T., Yanai, N. \& Kitagawa, S. Polymerization reactions in porous coordination polymers. Chem. Soc. Rev. 38, 1128-1236 (2009).

16. Uemura, T. et al. Unveiling thermal transitions of polymers in subnanometre pores. Nat. Commun. 83, 1-4 (2010).

17. Uemura, T. et al. Conformation and molecular dynamics of single polystyrene chain confined in coordination nanospace. J. Am. Chem. Soc. 130, 6781-6788 (2008).

18. Wu, C. G. \& Bein, T. Conducting polyaniline filaments in a mesoporous channel host. Science 264, 1757-1759 (1994).

19. Wu, C. G. \& Bein, T. Conducting carbon wires in ordered, nanometer-sized channels. Science 266, 1013-1015 (1994). 
20. Kageyama, K., Tamazawa, J. \& Aida, T. Extrusion polymerization: catalyzed synthesis of crystalline linear polyethylene nanofibers within a mesoporous silica. Science 285, 2113-2115 (1999).

21. Chelazzi, D., Ceppatelli, M., Santoro, M., Bini, R. \& Schettino, V. High pressure synthesis of crystalline polyethylene using optical catalysis. Nat. Mater. 3, 470-475 (2004).

22. Citroni, M., Ceppatelli, M., Bini, R. \& Schettino, V. Laser-induced selectivity for dimerization versus polymerization of butadiene under pressure. Science 295, 2058-2060 (2002)

23. Schettino, V. \& Bini, R. Molecules under extreme conditions: chemical reactions at high pressure. Phys. Chem. Chem. Phys. 5, 1951-1965 (2003).

24. Ciabini, L., Santoro, M., Gorelli, F. A., Bini, R., Schettino, V. \& Raugei, S. Triggering dynamics of the high-pressure benzene amorphization. Nat. Mat 6, 39-43 (2007).

25. Haines, J. et al. Topologically ordered amorphous silica obtained from the collapsed siliceous zeolite, silicalite-1-F: a step forward 'perfect glasses'. J. Am. Chem. Soc. 131, 12333-12338 (2009).

26. Haines, J., Cambon, O., Levelut, C., Santoro, M., Gorelli, F. \& Garbarino, G. Deactivation of pressure induced amorphization in silicalite $\mathrm{SiO}_{2}$ by insertion of guest species. J. Am. Chem. Soc. 132, 8860-8861 (2010).

27. Coasne, B. et al. Enhanced mechanical strength of zeolites by adsorption of guest molecules. Phys. Chem. Chem. Phys. 13, 20096-20099 (2011).

28. Santoro, M., Gorelli, F., Haines, J., Cambon, O., Levelut, C. \& Garbarino, G. Silicon carbonate phase formed from carbon dioxide and silica under pressure. Proc. Natl Acad. Sci. USA 108, 7689-7692 (2011).

29. Smit, B. \& Siepmannt, J. I. Simulating the adsorption of alkanes in zeolites. Science 264, 1118-1120 (1994).

30. Smit, B. \& Maesen, T. L. M. Commensurate 'freezing' of alkanes in the channels of a zeolites. Nature 374, 42-44 (1995).

31. Chelazzi, C., Ceppatelli, C., Santoro, M., Bini, R. \& Schettino, V. Pressureinduced polymerization in solid ethylene. J. Phys. Chem. B 109, 21658-21663 (2005).

32. Zvereva-Loëte, N., Ballandras, A., Weber, G., Rotger, M., Boudon, V. \& Simon, J. M. Experimental IR study and ab initio modelling of ethylene adsorption in a MFI-type host zeolite. Mol. Phys. 107, 2081-2093 (2009).

33. Fontana, L. et al. High-pressure crystalline polyethylene studied by x-ray diffraction and $a b$ initio simulations. Phys. Rev. B 75, 174112 (2007).

34. Birch, F. Finite elastic strain of cubic crystals. Phys. Rev. 71, 809-824 (1947).

35. Angel, R. J., Allan, D. R., Miletich, R. \& Finger, L. W. The use of quartz as an internal pressure standard in high-pressure crystallography. J. Appl. Crystallogr. 30, 461 (1997).

36. Gorelli, F. A., Ulivi, L., Santoro, M. \& Bini, R. The $\varepsilon$ phase of solid oxygen: evidence of an $\mathrm{O}_{4}$ molecule lattice. Phys. Rev. Lett. 83, 4093-4096 (1999).

37. Hammersley, A. P., Svensson, S. O., Hanfland, M., Fitch, A. N. \& Häusermann, D. Two-dimensional detector software: from real detector to idealised image or two-theta scan. High Press. Res. 14, 235-248 (1996).

38. Angel, R. J. Absorption corrections for diamond-anvil pressure cells implemented in a software package Absorb-6.0. J. Appl. Crystallogr. 37, 486-492 (2004)
39. Sheldrick, G. M. A short history of SHELX. Acta Crystallogr. A64, 112-122 (2008).

40. Mao, H. K., Bell, P. M., Shaner, J. V. \& Steinberg, D. J. Specific volume measurements of $\mathrm{Cu}, \mathrm{Mo}, \mathrm{Pd}$, and $\mathrm{Ag}$ and calibration of the ruby $R_{1}$ fluorescence pressure gauge from 0.06 to 1Mbar. J. Appl. Phys. 49, 3276-3283 (1978).

41. Ghiorso, M. S., Carmichael, I. S. E. \& Moret, L. K. Inverted high-temperature quartz unit cell parameters and properties of the $\alpha-\beta$ inversion. Contrib. Mineral. Petrol. 68, 307 (1979).

42. Carpenter, M. A., Salje, E. K. H., Graeme-Barber, A., Wruck, B., Dove, M. T. \& Knight, K. S. Calibration of excess thermodynamic properties and elastic constant variations associated with the alpha-beta phase transition in quartz. Am. Mineral 83, 2-22 (1998).

\section{Acknowledgements}

We acknowledge the support from the European Union (LENS Contract FP7 G.A.No. 228334 LASERLABEUROPE), the Ente Cassa di Risparmio di Firenze, the Deep Carbon Observatory initiative (Grant N. 2011-10-01 from the Alfred P. Sloan Foundation for the project entitled 'Physics and Chemistry of Deep Carbon Bearing Fluids and Mineral'), and the Agence Nationale de la Recherche (Contract ANR-09-BLAN-0018-01).

\section{Author contributions}

M.S. proposed the research, did the project planning, the high-pressure synthesis of PESIL, and performed the optical spectroscopy measurements; he contributed to powder $\mathrm{XRD}$ measurements, data analysis and interpretation, and wrote the paper. F.A.G. contributed to powder XRD measurements, data analysis and interpretation. R.B. contributed to data analysis, interpretation and to writing the paper. J.H. and A. vd L. performed the single-crystal XRD measurements, the single-crystal XRD data analysis and interpretation, and contributed to writing the paper.

\section{Additional information}

Accession codes: the X-ray crystallographic coordinates for structures reported in this article have been deposited at the Cambridge Crystallographic Data Centre (CCDC), under deposition number CCDC 919628. These data can be obtained free of charge from The Cambridge Crystallographic Data Centre via www.ccdc.cam.ac.uk/data_request/cif.

Supplementary Information accompanies this paper at http://www.nature.com/ naturecommunications

Competing financial interests: The authors declare no competing financial interests.

Reprints and permission information is available online at http://npg.nature.com/ reprintsandpermissions/

How to cite this article: Santoro, M. et al. High-pressure synthesis of a polyethylene/ zeolite nano-composite material. Nat. Commun. 4:1557 doi: 10.1038/ncomms2564 (2013). 